
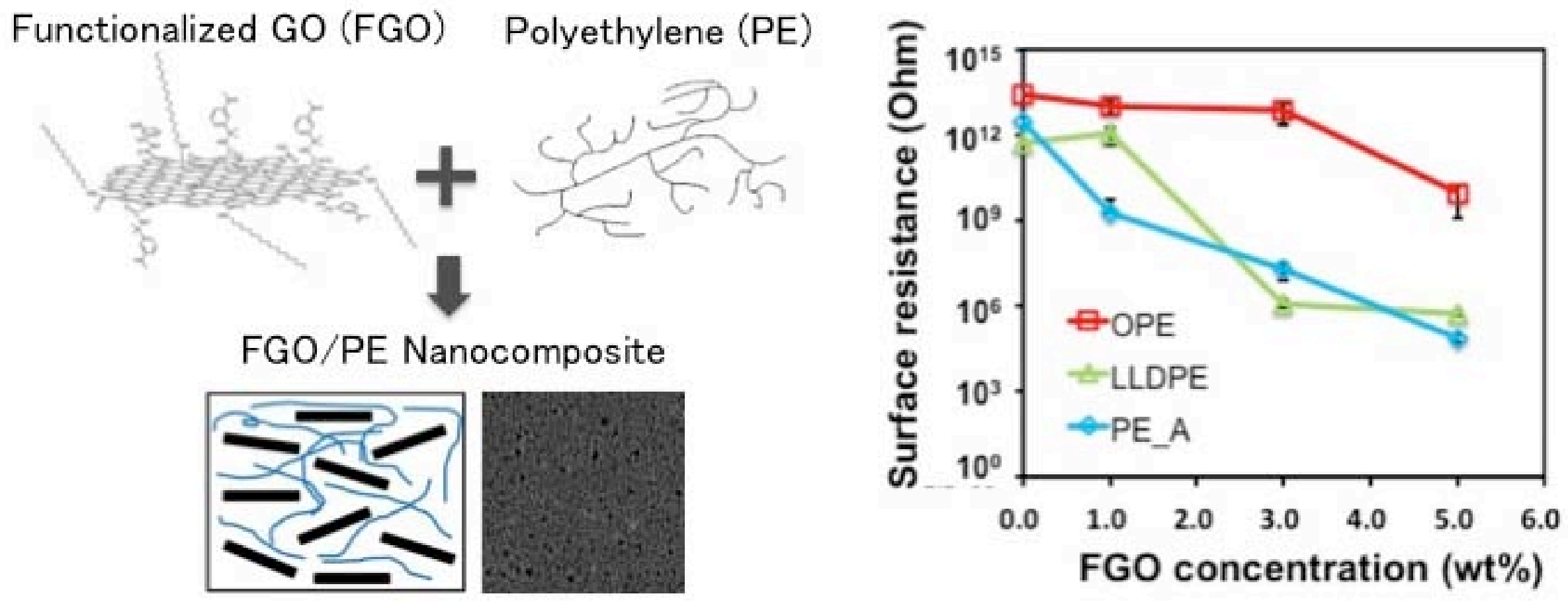


\title{
Graphene-Polyethylene Nanocomposites: Effect of Graphene Functionalization
}

\author{
Saibom Park ${ }^{\mathrm{a}, \mathrm{c}}$, Siyao He ${ }^{\mathrm{b}}$, Jianeng Wang ${ }^{\mathrm{a}}$, Andreas Stein ${ }^{\mathrm{b}}$ and Christopher W. \\ Macosko $^{\mathrm{a}^{*}}$ \\ ${ }^{a}$ Department of Chemical Engineering and Material Science, University of Minnesota, \\ Minneapolis, MN 55455-0331, USA \\ ${ }^{b}$ Department of Chemistry, University of Minnesota, Minneapolis, MN 55455-0431, USA \\ ${ }^{c}$ Present address: LG Chem Ltd., Research Park, Daejeon, 34122, Republic of Korea
}

\begin{abstract}
The properties of polymer nanocomposites depend strongly on how well nanoparticles are dispersed. However, the hydrophobic nature and low polarity of PE have made effective dispersion of nano-filler difficult without compatibilization. We have found improved dispersion of chemically functionalized graphene oxide (FGO) in PE. The hydroxyl and epoxide groups on graphene oxide (GO) are sites for grafting on functional groups like alkanes. We covalently attach alkyl chains and benzyl moieties to GO. This FGO was solvent blended into three types of polyethylene: high density, linear low density, and oxidized PE matrices. Visual observation of cast films and optical micrographs revealed that FGO was more homogeneously dispersed than unmodified GO. Simple thermal reduction of the FGO sheets dispersed in PE at $210{ }^{\circ} \mathrm{C}$ achieved a $10^{6}$-fold reduction in electrical resistance, much greater than that of other unmodified graphene composites. Moreover the tensile modulus of the low density PE was improved 12-fold and that of the high density PE 3-fold with 5\% FGO.
\end{abstract}

Keywords: Graphene, Polyethylene, Polymer nanocomposites 


\section{Introduction}

Electrically conductive composites composed of polymers and conducting fillers have been broadly studied over the past few decades $[1,2,3]$. These materials can be employed as antistatic or electromagnetic shielding materials to avoid damage to electronics by electrostatic discharge or electromagnetic interference. Polyethylene (PE) is a low-cost, general-purpose polymer which shows good mechanical properties and high chemical resistance. It can be used for numerous applications, for example, as a packaging material for electronics, if it is modified to have enhanced electrical conductivity.

Conventional conductive fillers are usually micrometer-scale metal powders or carbonaceous materials such as carbon black. In order to reach the percolation threshold with these fillers, the filler content needs to be as high as 10-50 wt $\%$ [4,5]. Such high levels result in poor mechanical properties, processing difficulties, and contamination of electronics due to sloughed off carbon black particles. Unlike traditional polymer composites, including micronscale fillers, the dispersion of carbon nanotubes (CNTs) into polymer systems offers a fascinating method to minimize the aforementioned problems. CNTs have significant benefits, i.e., high aspect ratio and excellent electrical conductivity, which in turn facilitate the development of conducting networks and thus convert the insulating polymer to a conducting composite at a low CNT content, often as low as $0.5 \mathrm{wt} \%[6,7,8,9]$. As for CNT/polymer functional composites, many distinctive properties of CNTs, such as electrical, thermal, optical and damping properties along with their excellent mechanical properties, are utilized to advance multi-functional composites for applications requiring heat resistance, chemical sensing, electrical and thermal management, photoemission, electromagnetic absorbtion and energy 
storage $[4,10,11]$. However, owing to the low yield and expensive production and purification process, the manufacturing expense of CNTs is still high, which limits commercial application of CNT-based conducting composites. Also dispersion of CNTs into polymers and orientation during processing remain challenging problems $[4,6.9]$.

Graphene has attracted much research attention due to its high aspect ratio, and desirable mechanical, electrical, and thermal properties $[1,2,3]$. Bulk production of graphene sheets from graphite oxide based on a chemical method may prove to be cheaper than production of CNTs $[12,13,14]$. These unique attributes make graphene attractive for applications in many technological areas, such as thermally and electrically conducting, reinforced polymer composites, electronic circuits, and transparent and flexible electrodes for displays $[1,2,3]$.

The challenge in creating graphene/polyethylene composites is that the low polarity of polyethylene leads to poor dispersion of graphene $[15,16]$. To overcome this, we start with graphene oxide (GO), which can be economically produced from graphite using strong acids [17]. GO is not electrically conductive but conductivity can be restored by heating at relatively low temperatures $[18,19]$. Graphite oxide is composed of covalently-attached hydroxyl, epoxy, carbonyl and carboxyl groups. The presence of these functional groups makes graphite oxide hydrophilic and easily swellable in water. Moreover, these functional groups provide means for covalent functionalization [20].

Here we report processing and properties of PE nanocomposites made with functionalized GO (FGO). Benzyl moieties and 18-carbon alkyl chains were attached to GO via urethane linkage and amide bond formation, respectively. The alkyl chains and benzyl moieties help GO to be exfoliated readily and lower the FGO polarity for better dispersion within PE. Linear low density PE (LLDPE), relatively high density PE (PE_A), and oxidized PE (OPE) 
were selected as polymer matrices. Dispersion of FGO in LLDPE, PE_A, and OPE was compared to the unfunctionalized GO and to GO which was thermally reduced (TRG) before dispersion in PE. We characterized these composites using optical microscopy and electrical and mechanical property measurements. We compared the conductivity of the FGO nanocomposites before and after reduction to determine the efficiency of thermal treatment at moderate temperatures.

\section{Experimental}

\subsection{Materials}

PE_A was obtained from Sigma-Aldrich (product \# 428108). It is a polymer containing 5-10 wt \% hexene co-monomer. LLDPE, provided by Dow Chemical, is a linear low density PE copolymer of ethylene and octene (ENGAGETM 8200 CAS 26221-73-8). OPE was purchased from Honeywell. It is an oxidized PE with $3.3 \mathrm{wt} \%$ oxygen content $\left(\mathrm{A}-\mathrm{C}^{\circledR} 656\right.$, oxidized PE homopolymer). Molecular characteristics of PE_A, LLDPE and OPE, including number averaged molecular weight $\left(\mathrm{M}_{\mathrm{n}}\right)$, polydispersity index (PDI), viscosity, density, melting point, composition and crystallinity are summarized in Table 1 . Values of $\mathrm{M}_{\mathrm{n}}$ for the three different PE were measured via gel permeation chromatography (GPC, PL-GPC 220 High Temperature Chromatograph) using trichlorobenzene as the eluent at $135^{\circ} \mathrm{C}$. TRG in this study was produced by thermal exfoliation of graphite oxide and kindly provided by Vorbeck Materials. Its properties are reported in reference [16]. Sodium nitrate (ACS grade), potassium permanganate (ACS grade), hydrogen peroxide (30\% solution in water, ACS grade), toluene (HPLC grade), and potassium bromide (IR grade) were obtained from Fisher Scientific. Octadecylamine (98\%), $N, N^{\prime}$-dimethylformamide, anhydrous (99.8\%), 3-isopropenyl- $\alpha, \alpha$-dimethylbenzylisocyanate 
(TMI, 95\%), 1,2-dichlorobenzene (ReagentPlus ${ }^{\circledR}$ Grade) and 1,4-diazabicyclo[2.2.2] octane (99\%), were purchased from Sigma-Aldrich. Ammonium hydroxide (28-30\% in water, ACS grade) and tert-butanol (99\%) were purchased from Macron. Sulfuric acid (98\%, ACS grade) and hydrochloric acid (37\%, ACS grade) were purchased from BDH. Graphite flakes (SP1 grade) were sourced from Bay Carbon. Deionized (DI) water with a resistivity of $18 \mathrm{M} \Omega \cdot \mathrm{cm}^{-2}$ was produced onsite using a Barnstead purification system.

Table 1. Molecular Characteristics of PEs.

\begin{tabular}{|c|c|c|c|c|c|c|c|}
\hline Sample code & $\begin{array}{c}\mathrm{M}_{\mathrm{n}} \\
\mathrm{g} / \mathrm{mol}\end{array}$ & PDI & $\begin{array}{c}\text { Viscosity } \\
\text { Pa•s }\end{array}$ & $\begin{array}{l}\text { Density } \\
\mathrm{g} / \mathrm{ml}\end{array}$ & $\begin{array}{l}\text { Melting point } \\
{ }^{\circ} \mathrm{C}\end{array}$ & Composition & Crystallinity \\
\hline $\begin{array}{l}\text { PE from Aldrich } \\
\qquad \text { (PE_A) }\end{array}$ & $9600^{\mathrm{a}}$ & 5.6 & $\begin{array}{l}200^{\mathrm{d}} \\
70^{\mathrm{e}}\end{array}$ & $0.93^{\mathrm{f}}$ & $122^{\mathrm{b}}$ & $\begin{array}{c}\text { Hexene } \\
5-10 \mathrm{wt} \%^{\mathrm{f}}\end{array}$ & $0.49^{\mathrm{c}}$ \\
\hline $\begin{array}{l}\text { EG8200 from Dow } \\
\text { (LLDPE) }\end{array}$ & $42000^{\mathrm{a}}$ & 3.0 & $\begin{array}{l}7300^{\mathrm{d}} \\
1200^{\mathrm{e}}\end{array}$ & $0.87^{\mathrm{f}}$ & $65^{\mathrm{b}}$ & $\begin{array}{l}\text { Octene } \\
7.3 \mathrm{wt}^{\mathrm{f}}{ }^{\mathrm{f}}\end{array}$ & $0.16^{\mathrm{c}}$ \\
\hline $\begin{array}{c}\text { Oxidized PE } \\
\text { from Honeywell } \\
\text { (OPE) }\end{array}$ & $450^{\mathrm{a}}$ & 10.2 & $\begin{array}{l}1.0^{\mathrm{d}} \\
0.8^{\mathrm{e}}\end{array}$ & $0.92^{\mathrm{f}}$ & $92^{\mathrm{b}}$ & $\begin{array}{l}\text { Oxygen } \\
3.3 w t \%\end{array}$ & $0.29^{c}$ \\
\hline $\begin{array}{l}{ }^{\mathrm{a}} \mathrm{GPC} \text {, trichlorobenze } \\
{ }^{\mathrm{b}} \mathrm{DSC} \text {, second heatin } \\
{ }^{\mathrm{c}} \text { Determined by divic } \\
{ }^{\mathrm{d}} \text { at } 140{ }^{\circ} \mathrm{C} \\
\mathrm{e}^{\mathrm{e}} \text { at } 210^{\circ} \mathrm{C} \\
{ }^{\mathrm{f}} \text { Data provided by su }\end{array}$ & $\begin{array}{l}\mathrm{RI} \text { de } \\
0{ }^{\circ} \mathrm{C} / \\
\text { the h } \\
\text { ier }\end{array}$ & $\begin{array}{l}\text { PS : } \\
\text { fusic }\end{array}$ & $\begin{array}{l}\text { dards, } \\
\text { y } 293 \mathrm{~J} / \mathrm{g}\end{array}$ & & & & \\
\hline
\end{tabular}

\subsection{Graphene synthesis and characterization}




\subsubsection{Synthesis of GO}

The method of GO synthesis was modified from Hummers' method [17]. In a typical synthesis, $2.5 \mathrm{~g} \mathrm{NaNO}_{3}$ was dissolved in $115 \mathrm{~mL}$ concentrated sulfuric acid in an ice bath, and 5 $\mathrm{g}$ of graphite was then added to the solution. Under moderate stirring, $5 \mathrm{~g} \mathrm{KMnO}_{4}$ was added every 10 minutes three times for $15 \mathrm{~g}$ total. The ice bath was then replaced by a room temperature water bath, and the mixture was allowed to react at $35{ }^{\circ} \mathrm{C}$ for $1 \mathrm{~h}$. Later, $230 \mathrm{~mL}$ of DI water was added to the reaction mixture and the temperature increased to $80^{\circ} \mathrm{C}$. After stirring for $15 \mathrm{~min}$, the mixture was further diluted to $1 \mathrm{~L}$. The reaction was quenched by adding hydrogen peroxide dropwise until the effervescence stopped and the mixture turned light brown. After overnight sedimentation, $600 \mathrm{~mL}$ of the supernatant was removed, and the rest of the mixture was divided into eight portions for purification.

To purify the GO, $40 \mathrm{~mL}$ of DI water was added to each portion with $1.5 \mathrm{~mL}$ concentrated hydrochloric acid. After stirring and shaking inside the centrifuge tube, the GO was spun down (Marathon 8k) at $3000 \mathrm{rpm}$ for $15 \mathrm{~min}$. This procedure was repeated for a total of 10 times to remove metal ions and sulfate ions (no precipitation in $0.1 \mathrm{M} \mathrm{BaCl}_{2}$ ). The resulting material was then dialyzed against DI water with cellulose dialysis tubing (Fisherbrand, MWCO: 6000-8000) until no precipitate was observed upon mixing the dialyzing solvent with $0.1 \mathrm{M}$ silver nitrate solution. The GO was then redispersed in DI water and neutralized with ammonium hydroxide. After $1 \mathrm{~h}$ sonication in a bath sonicator (Branson 3510 ultrasonic cleaner, Emerson Electric Co.), the GO dispersion was centrifuged at $1500 \mathrm{rpm}$ for $10 \mathrm{~min}$ to remove any poorly oxidized graphite. The supernatant was collected as the GO stock solution $(2 \mathrm{mg} / \mathrm{mL})$. Later, the stock solution was freeze-dried (Freezemobile, SP Scientific) to obtain the solid GO for further modification. 


\subsubsection{Synthesis of TMI/octadecylamine-functionalized GO (FGO)}

To synthesize functionalized GO, $300 \mathrm{mg}$ of the dried $\mathrm{GO}$ was dispersed in $75 \mathrm{~mL}$ anhydrous $N, N^{\prime}$-dimethylformamide (DMF) and then sonicated in a bath sonicator (Branson 3510) for $1 \mathrm{~h}$. The resulting homogenous dispersion was transferred into a $150 \mathrm{~mL}$ two-necked round bottom flask and 1,4-diazabicyclo[2.2.2] octane (DABCO, $5 \mathrm{mg}$ per $100 \mathrm{mg} \mathrm{GO}$ ) was added as the catalyst. The dispersion was purged under nitrogen flow for $2 \mathrm{~h}$ with $300 \mathrm{rpm}$ stirring. After that, $6 \mathrm{~mL}$ of 3-isopropenyl- $\alpha, \alpha$-dimethylbenzylisocyanate (TMI) was injected and the mixture was allowed to react at $60{ }^{\circ} \mathrm{C}$ for $24 \mathrm{~h}$ under nitrogen. The reaction was quenched by adding $225 \mathrm{~mL}$ dry toluene, and the resulting mixture was then centrifuged at $3000 \mathrm{rpm}$ for 15 minutes. The precipitate from the centrifugation was washed 3 times with toluene and twice with tert-butanol followed by freeze drying to obtain the intermediate product (TMI-GO).

The resulting TMI functionalized GO was dispersed in DMF in the same way as described before ( $2 \mathrm{mg} / \mathrm{mL})$. The octadecylamine was then added (4 mg per $1 \mathrm{mg} \mathrm{GO})$ and the reaction was kept at $70{ }^{\circ} \mathrm{C}$ for $24 \mathrm{~h}$ while stirring at $300 \mathrm{rpm}$. The reaction was terminated by pouring the mixture into ethanol. The functionalized GO flocculated immediately. The crude product was collected by centrifugation and purified by 4 washes in dry ethanol and 2 washes in DI water. The purified product, TMI-octadecyl-GO, referred to hereafter as FGO, was freeze dried to obtain a powder. Figure 1 illustrates the steps of the FGO synthesis. TMI modification is believed to help exfoliation of GO sheets in the organic solvent, which in turn benefits the subsequent octadecyl functionalization. Also, the unsaturated moiety in the TMI molecule may be able to bond to PE chains at elevated temperature to improve the particle-matrix adhesion. 

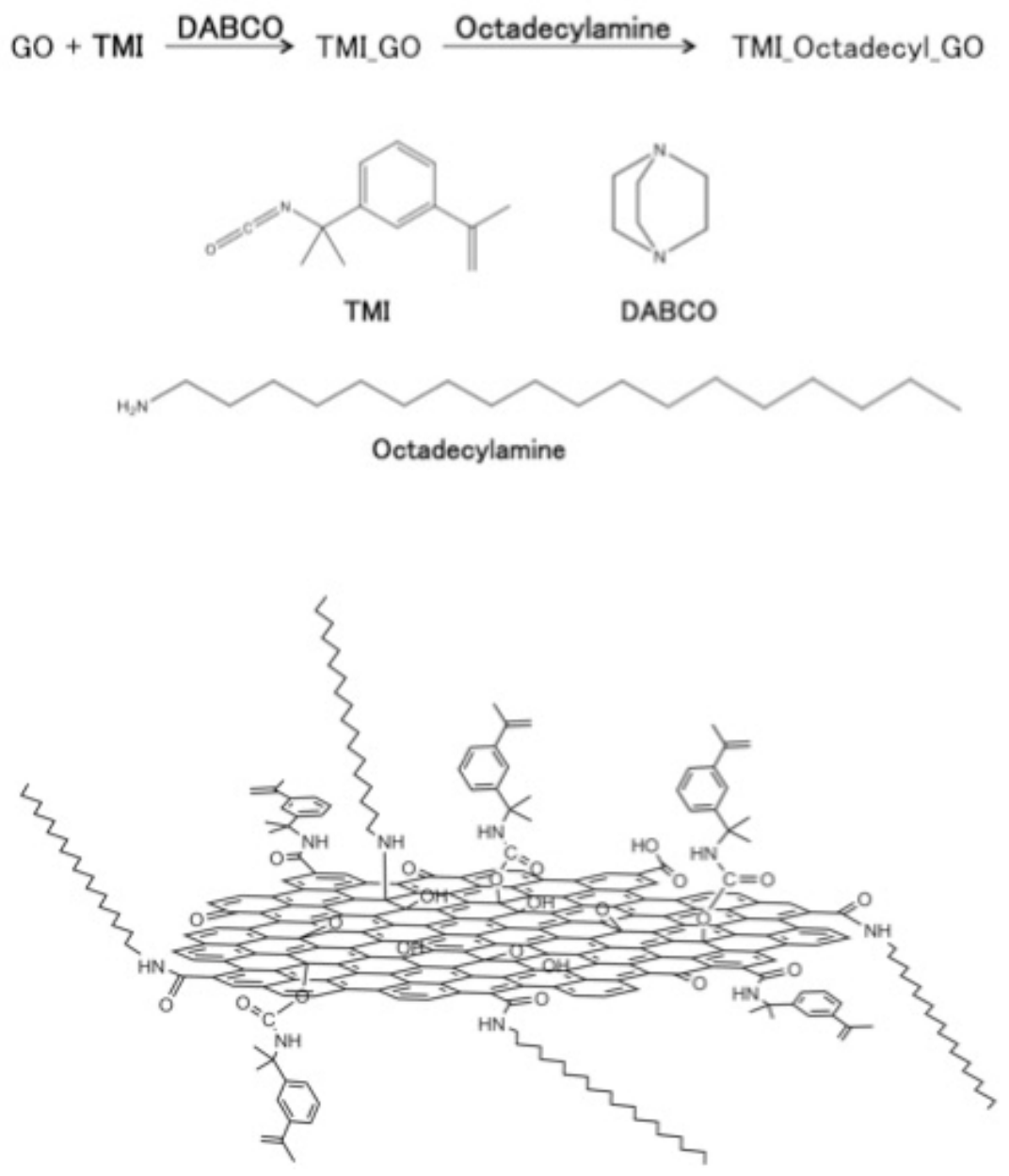

Fig. 1. Synthesis and schematic structure of FGO.

\subsubsection{Characterization}

The viscosity of PE was measured using parallel-plate frequency sweep tests at $140{ }^{\circ} \mathrm{C}$ and $210{ }^{\circ} \mathrm{C}$ with an ARES rheometer (TA instrument). The melting point and degree of crystallinity of PE were determined by differential scanning calorimetry (DSC, TA Instruments Q1000) using TA Universal Analysis software. 4.0-6.0 mg of PE was loaded into hermetic aluminum pans. The scanning procedure was performed from $40{ }^{\circ} \mathrm{C}$ to $150{ }^{\circ} \mathrm{C}$ with a heating/cooling rate of $10^{\circ} \mathrm{C} / \mathrm{min}$. Crystallinity was calculated by dividing the heat of fusion 
obtained from integrating the area of the melting endothermic peak by $293 \mathrm{~J} / \mathrm{g}$ [21], the heat of the fusion for a $100 \%$ crystalline PE.

X-ray photoelectron spectroscopy (XPS, SSX-100 system, Surface Science Laboratories Inc., equipped with a monochromated $\mathrm{Al} \mathrm{K} \alpha \mathrm{X}$-ray source) was applied to estimate the atomic percentage of carbon and oxygen for GO and FGO. The analysis spot size of the scan was $1 \mathrm{x} 1$ $\mathrm{mm}^{2}$ and the survey spectrum was collected using 12-16 scans. Carbon-to-oxygen ratios were calculated from the deconvoluted XPS spectra (Fig. S1). The atomic carbon-to-oxygen percentage was 2:1 and 8:1 for GO and FGO, respectively.

Transmission infrared (IR) absorbance spectra of GO, FGO and TRG were obtained using a Fourier-transform-IR (FT-IR) spectrometer (Magna-FTIR 760, Nicolet). The spectra were collected from $4000 \mathrm{~cm}^{-1}$ to $400 \mathrm{~cm}^{-1}$. Wide-angle X-ray diffraction patterns of GO, FGO and TRG were collected with an X-ray diffractometer (Bruker-AXS D5005) using CuKa radiation as a source at $45 \mathrm{kV}, 40 \mathrm{~mA}$ and the $2 \theta$ range of $4-20^{\circ}$. Transmission electron microscopy (TEM, FEI, Tecnai T12) images were obtained to survey the sizes and morphologies of the FGO and TRG sheets. Each sample was stirred in ethanol and sonicated with a bath sonicator for $5 \mathrm{~min}$. Lacey carbon TEM grids were dipped into the dispersions and allowed to air-dry before imaging.

\subsection{Dispersion}

Graphene/PE nanocomposites were prepared by solvent blending with three GO samples (untreated GO, FGO and TRG) and three PE samples (PE_A, LLDPE, and OPE). Graphene was dispersed into PE using 1,2-dichlorobenzene (DCB). Up to $50 \mathrm{mg}$ of graphene was added to 10 $\mathrm{mL}$ of $\mathrm{DCB}$ and stirred for $48 \mathrm{~h}$ at room temperature. About $1.0 \mathrm{~g}$ of PE solid was then added, 
and the mixture was heated in an oil bath at $\sim 130{ }^{\circ} \mathrm{C}$ for at least $2 \mathrm{~h}$. Graphene/PE mixtures in DCB were then cast on glass plates, forming composite films with the compositions of 1.0, 3.0 and $5.0 \mathrm{wt} \%$. After the film casting, solvent removal continued for at least $48 \mathrm{~h}$ at room temperature and another $72 \mathrm{~h}$ in a vacuum oven $\left(24{ }^{\circ} \mathrm{C}, 5 \mathrm{mbar}\right)$. These films were annealed at $210^{\circ} \mathrm{C}$ in an oven for $1 \mathrm{~h}$. Some samples were treated with different thermal reduction times, annealed for 0,5 , and $30 \mathrm{~min}$. Figure 2 illustrates the steps of the dispersion process.

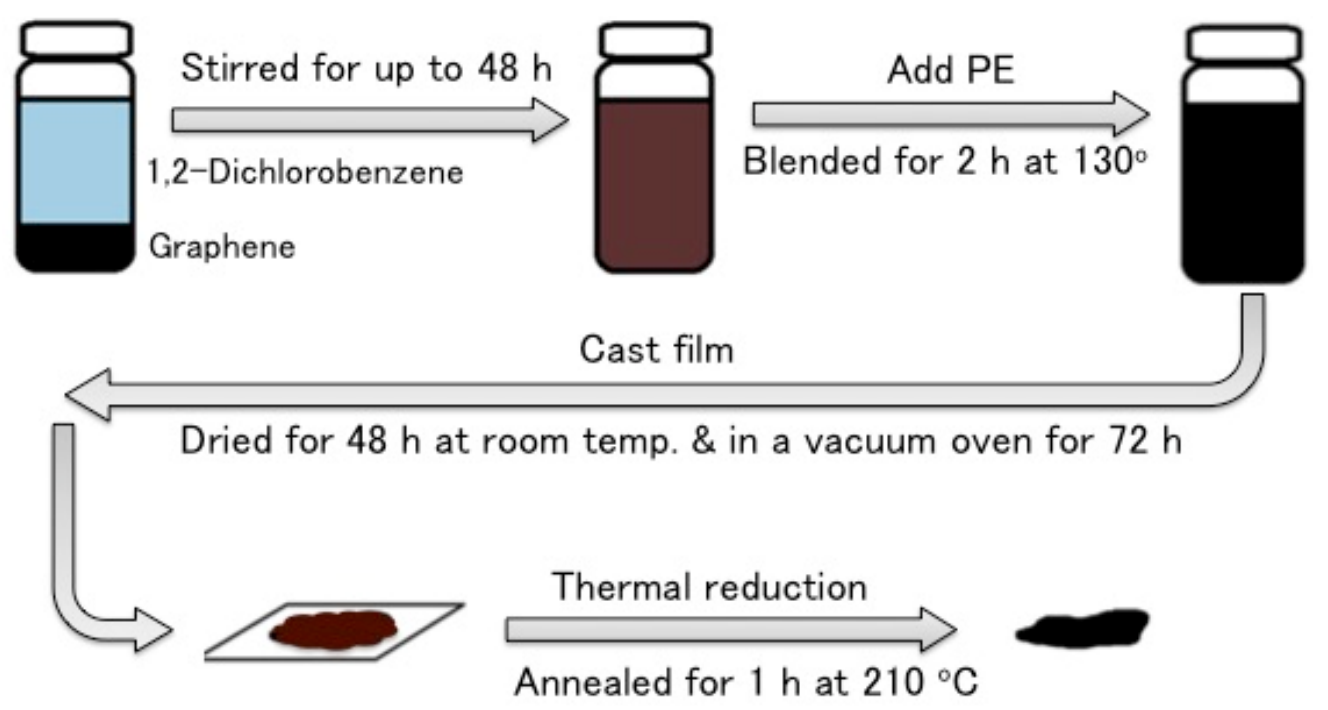

Fig. 2. Schematic diagram of preparation of graphene/PE nanocomposites.

\subsection{Characterization of nanocomposites}

Optical microscopy was applied to investigate the dispersion of graphene in PE. Approximate $1 \mathrm{mg}$ of composite was loaded between two glass slides placed in an oven at $130{ }^{\circ} \mathrm{C}$ for 2 minutes which caused it to melt. It was then compressed to a thickness of about 100 $\mu \mathrm{m}$ measured with a digital micrometer (Mitutoyo). Optical microscopy images were taken (Eclipse Ti, Nikon) and thresholded to create black and white images using ImageJ software. 
Tensile moduli of the composites were measured with an RSA-G2 (TA instruments). Composite samples were cut to rectangular strips $4-6 \mathrm{~mm}$ in width, then clamped between two film fixtures of the rheometer. The displacement rate of the sample was $0.01 \mathrm{~mm} / \mathrm{s}$, and the modulus was evaluated based on the slopes of stress-strain responses from $0.05-0.25 \%$ strain.

Surface resistance was measured using an 11-probe meter (PRS-801, Prostat). The conductivity was reported from the geometric average which was estimated based on 3 to 4 different spots of a film sample to ensure the uniformity of samples. Crystallinity was measured using differential scanning calorimetry (DSC, Q1000 TA Instruments) by heating at $10{ }^{\circ} \mathrm{C} / \mathrm{min}$ from ambient to $200^{\circ} \mathrm{C}$.

\section{Results and discussion}

The properties of polymer nanocomposites depend strongly on how well nano-fillers are dispersed in the polymer matrix. In particular, the hydrophobic nature and low polarity of PE have made effective dispersion of graphene difficult without compatibilization. We were able to improve dispersion through chemically functionalizing GO. The hydroxyl and epoxide groups on GO are sites for grafting on functional groups like alkanes. We covalently attached benzyl moieties by TMI modification and 18-carbon alkyl chains to GO via amide bond formation. TMI modification via urethane linkage helps exfoliation of GO sheets in the organic solvent, which benefits the subsequent octadecyl functionalization. Also, the unsaturated moiety in TMI molecules may be able to bond to PE chains at elevated temperature to improve the particlematrix adhesion. In addition, the long alkyl chains attached to GO sheets can lower the polarity and increase the hydrophobicity of GO, leading to better compatibilization with PE. 


\subsection{Characterization of FGO sheets}

TEM images indicate that the ultrathin sheets of TRG and FGO have paper-like structures (Fig. 3a and b). In general, TRG shows more wrinkles than FGO. This has been attributed to the thermal reduction process with the elimination of oxygen forming mesopores and crumple voids [22,23]. Unlike functional groups such as hydroxyls or carboxyls, epoxy groups generate strain on the $\mathrm{C}-\mathrm{C}$ bond of the three-membered epoxide ring. When several epoxy groups line up, the sheet can form a kink that partially releases this strain energy and makes this configuration energetically favored. The selected area electron diffraction (SAED) patterns of FGO show only weak and diffuse rings, indicating that the FGO is multilayered. The diffraction spots of TRG suggest that it consists of single or a few layers, in agreement with the observed wrinkled morphology.

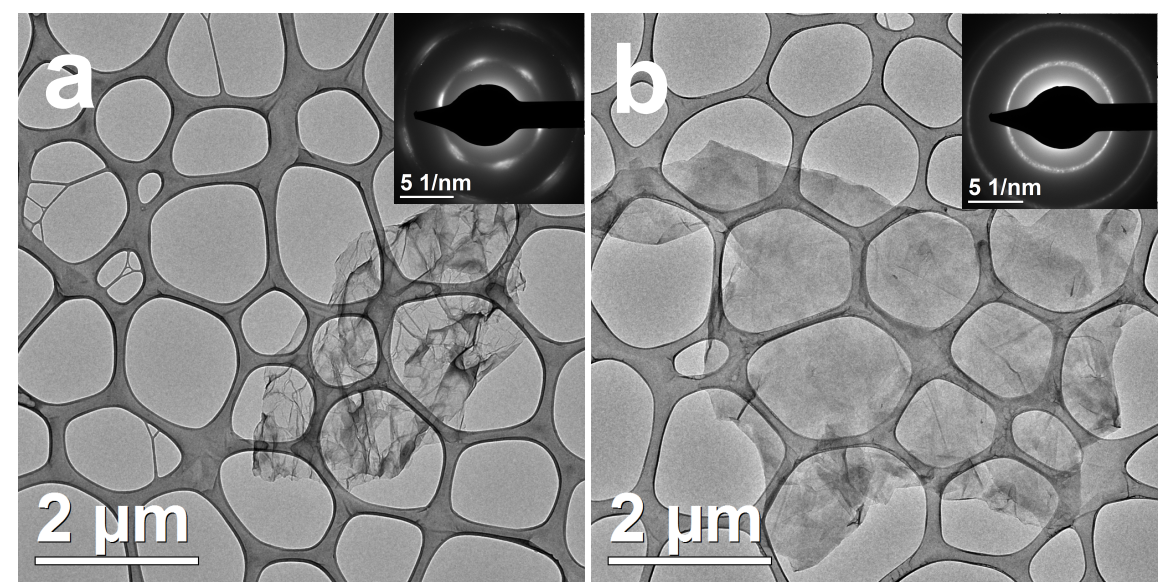

Fig. 3. TEM micrographs and electron diffraction patterns (insets) of (a) TRG and (b) FGO.

The functionalization of GO was confirmed by IR spectroscopy (Fig. 4a). The absorption peaks at 2920 and $2850 \mathrm{~cm}^{-1}$ in FGO represent the antisymmetric and symmetric stretching vibrations, respectively, of methylene groups in the long alkyl chains grafted onto GO [24]. The corresponding methylene scissoring and rocking bands appear at 1460 and $721 \mathrm{~cm}^{-1}$, respectively. 
Absorptions due to amide $\mathrm{C}=\mathrm{O}$ stretching and amide $\mathrm{N}-\mathrm{H}$ bending vibrations at 1650 and 1580 $\mathrm{cm}^{-1}$, respectively, and the absence of the carboxylate peak that was present in the original GO $\left(1721 \mathrm{~cm}^{-1}\right)$, confirm the amide linkage between the long-chain alkyl amine and the GO surface. Peaks at 1105 and $1070 \mathrm{~cm}^{-1}$ in the FGO spectrum can be ascribed to mixed skeletal modes of $\mathrm{C}-\mathrm{C}$ and $\mathrm{C}-\mathrm{O}$ bonds in $\mathrm{GO}[24,25]$. The peak at $1236 \mathrm{~cm}^{-1}$ is attributed to epoxide groups and that at $1370 \mathrm{~cm}^{-1}$ to bending of $\mathrm{C}-\mathrm{OH}$ phenolic groups in $\mathrm{GO}$ [25]. The presence of these absorption peaks indicates that not all available oxygen groups on GO have been utilized in the functionalization process. Because of the relatively low density of TMI functional groups on the FGO, as well as the overlapping of its characteristic absorption peaks with those of alkyl groups and GO, we cannot observe IR absorptions corresponding to TMI in the spectrum. A broad peak between 3700 and $3000 \mathrm{~cm}^{-1}$, which is apparent in all three spectra, arises from the stretching vibration of $\mathrm{OH}$ groups of water molecules adsorbed on $\mathrm{GO}$.

The structural change in FGO was observed by X-ray diffraction as shown in Fig. 4b. Upon functionalization, the (001) diffraction peak of GO located at $\sim 13^{\circ} 2 \theta$ shifts to $\sim 5^{\circ} 2 \theta$, indicating an increase of the interlayer spacing from $\sim 0.72 \mathrm{~nm}$ to $\sim 1.61 \mathrm{~nm}$ due to the incorporation of long alkyl functional groups. TRG does not display any prominent diffraction peaks in the $2 \theta$ range from 4 to $20^{\circ}$ due to more complete exfoliation, in agreement with previous characterization of TRG [16]. The lack of X-ray diffraction peaks signifies loss of the periodic order in GO layer exfoliation and agrees with the TEM observation of one or a few layers in TRG. After treating FGO for $1 \mathrm{~h}$ at $210{ }^{\circ} \mathrm{C}$ under $\mathrm{N}_{2}$, X-ray diffraction patterns showed more exfoliation and IR spectra show loss of the dodecyl groups, Figure S2. 


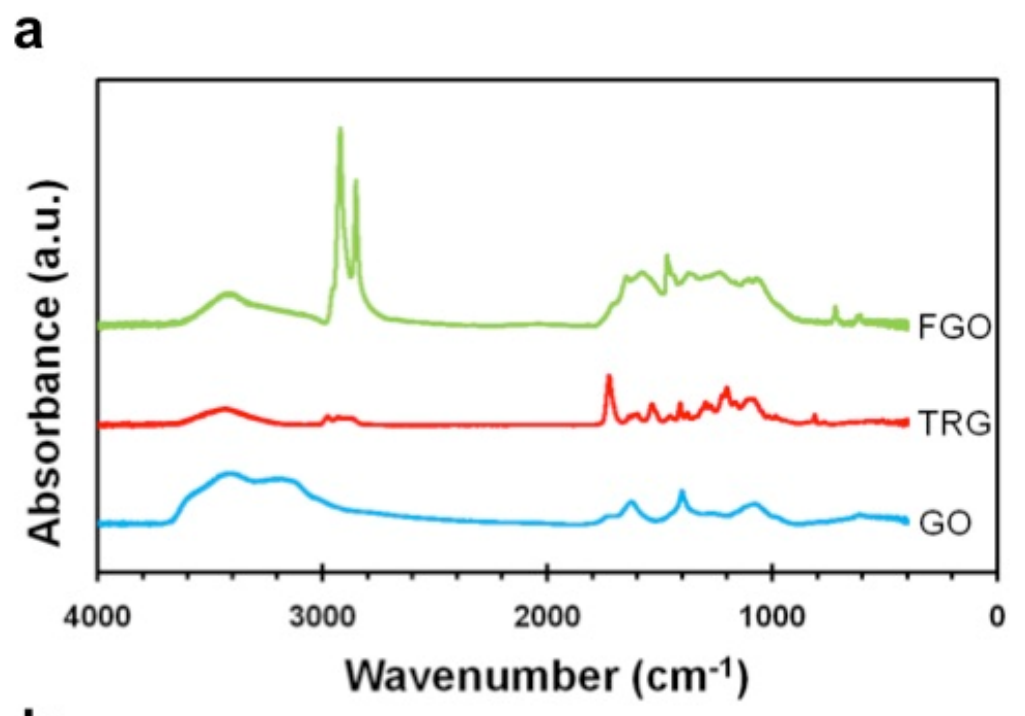

b

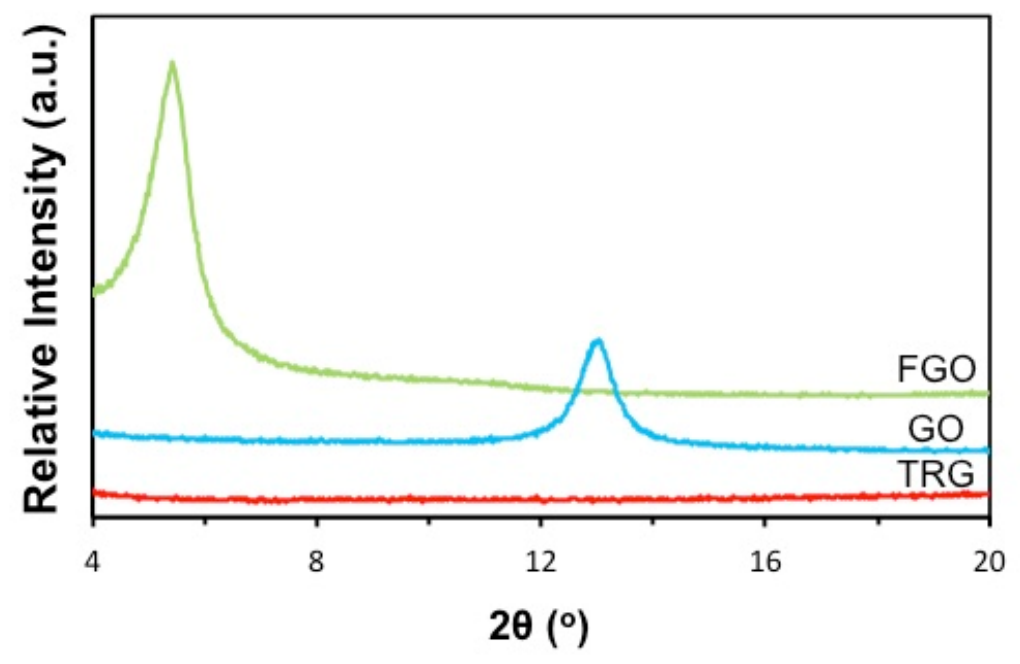

Fig. 4. (a) Transmission FT-IR absorbance spectra of FGO, GO and TRG. (b) Wide-angle X-ray diffraction (XRD) patterns of FGO, GO and TRG.

\subsection{Dispersion of FGO in PE}

In addition to the thermodynamic compatibility of filler sheets with polymers, processing has a substantial impact on the dispersion and properties of graphene reinforced polymer composites. Due to the intrinsic low miscibility between PE and graphene, restricted material 
diffusion and compressive flow fields in high viscosity polymer melts may result in reduced macroscopic distribution in melt compounded samples [16]. Therefore solution blending was employed in this study as it facilitated material transport in low viscosity solvents [16,26].

The crystallinity of FGO/PE nanocomposites was obtained from DSC analysis. The degree of crystallization slightly decreased at $3 \mathrm{wt} \%$ FGO loading in PE compared to pure PE (Table S1). We speculate that the large amount of FGO and its good dispersion significantly hinders the diffusion of polymer chains to the growing crystallites. We also did XPS analysis of FGO/PE composites before and after thermal treatment as well as of FGO. There was no peak shift after blending FGO with PE and even after thermal treatment (Fig. S3). This indicates that there are no chemical bonding or strong interaction between FGO and PE.

Optical microscopic images of the different composites reveal the morphology of FGO and TRG dispersed in different PE matrices. Images of PE_A films containing TRG and FGO with 1 and 3 wt\% are shown in Figure 5. The morphology of $1 \mathrm{wt} \%$ TRG in the PE_A matrix can be described as partially exfoliated with significant occurrence of graphene aggregation (Fig. 5a). The strong segregation of $3 w t \%$ TRG from PE_A is clearly observed (Fig. 5b). Apparent localization of TRG aggregates signifies phase separation due to intrinsic incompatibility. However, macroscopic distribution of FGO is significantly improved in PE_A (Fig. 5c and 5d). Enhanced FGO dispersion was also found in LLDPE and OPE matrices (see Supplementary Material). The functionalization of GO appears to be critical for the generation of evenly distributed graphene in polyethylene. 


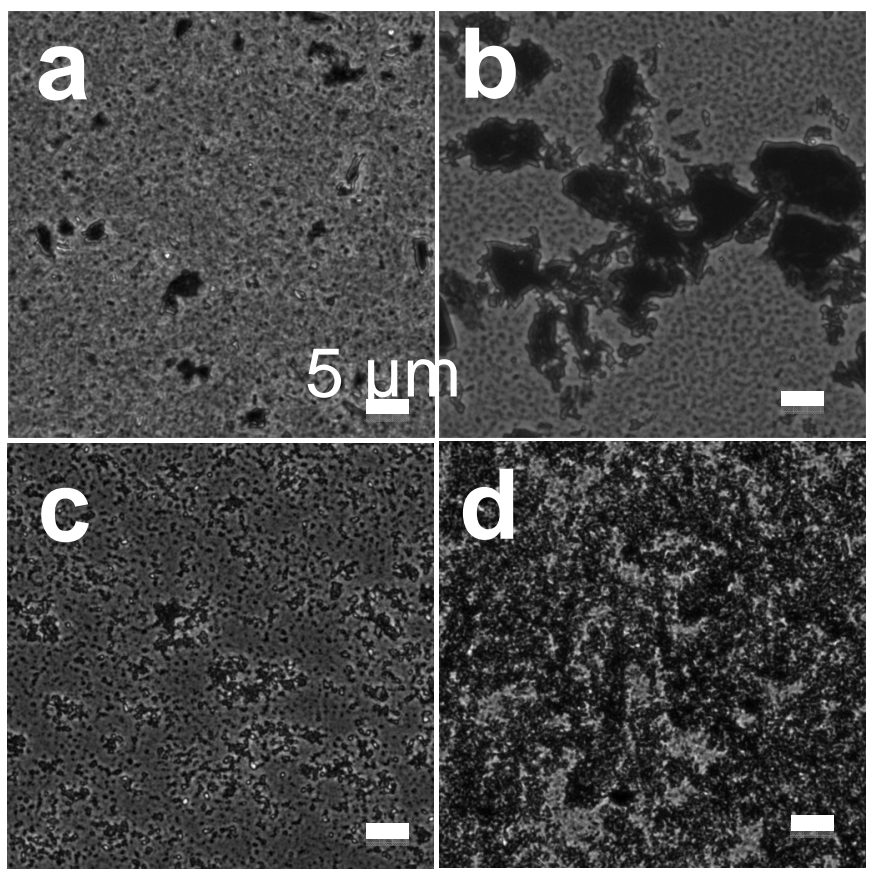

Fig. 5. Optical microscopy images of PE_A with (a) $1 \mathrm{wt} \% \mathrm{TRG}$, (b) $3 \mathrm{wt} \% \mathrm{TRG}$, (c) $1 \mathrm{wt} \%$ FGO, and (d) $3 \mathrm{wt} \%$ FGO.

Figure $6 \mathrm{a}$ and $6 \mathrm{~b}$ compare the dispersion of LLDPE with $3 \mathrm{wt} \%$ of TRG and FGO, respectively, after adjusting the color threshold. From images like these, relative dispersion indices were calculated. The dispersion index results, defined as the area occupied with the filler divided by total area, are shown in Figure 6c. A high index indicates a more homogeneous dispersion because the fillers uniformly cover the whole surface of the microscopic samples. Judging from these thresholded images, FGO is well dispersed throughout all PE matrices. In contrast, TRG is not homogeneously dispersed into PE, leading to a comparatively low dispersion index. This verifies the compatibilization of the FGO/PE blends by GO functionalization. In addition, OPE and LLDPE seem to lead to better dispersion with TRG and FGO than PE_A. The effect of different PEs on dispersion and on electrical conductivity does not coincide because the specific properties of PE also can affect the electrical conductivity of 
the composites. The relation between morphological difference and differences in electrical conductivity are described in the next section.

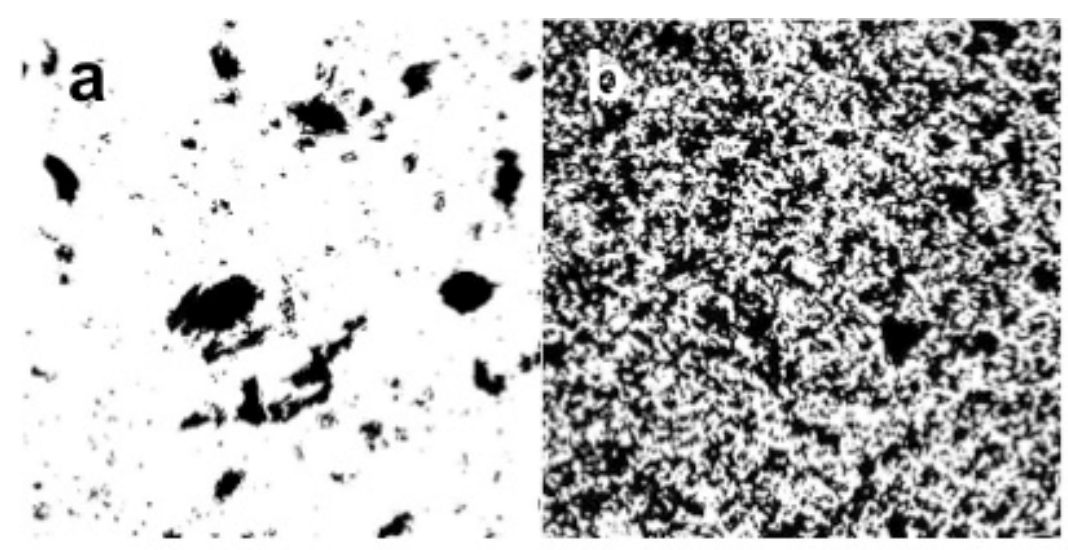

C

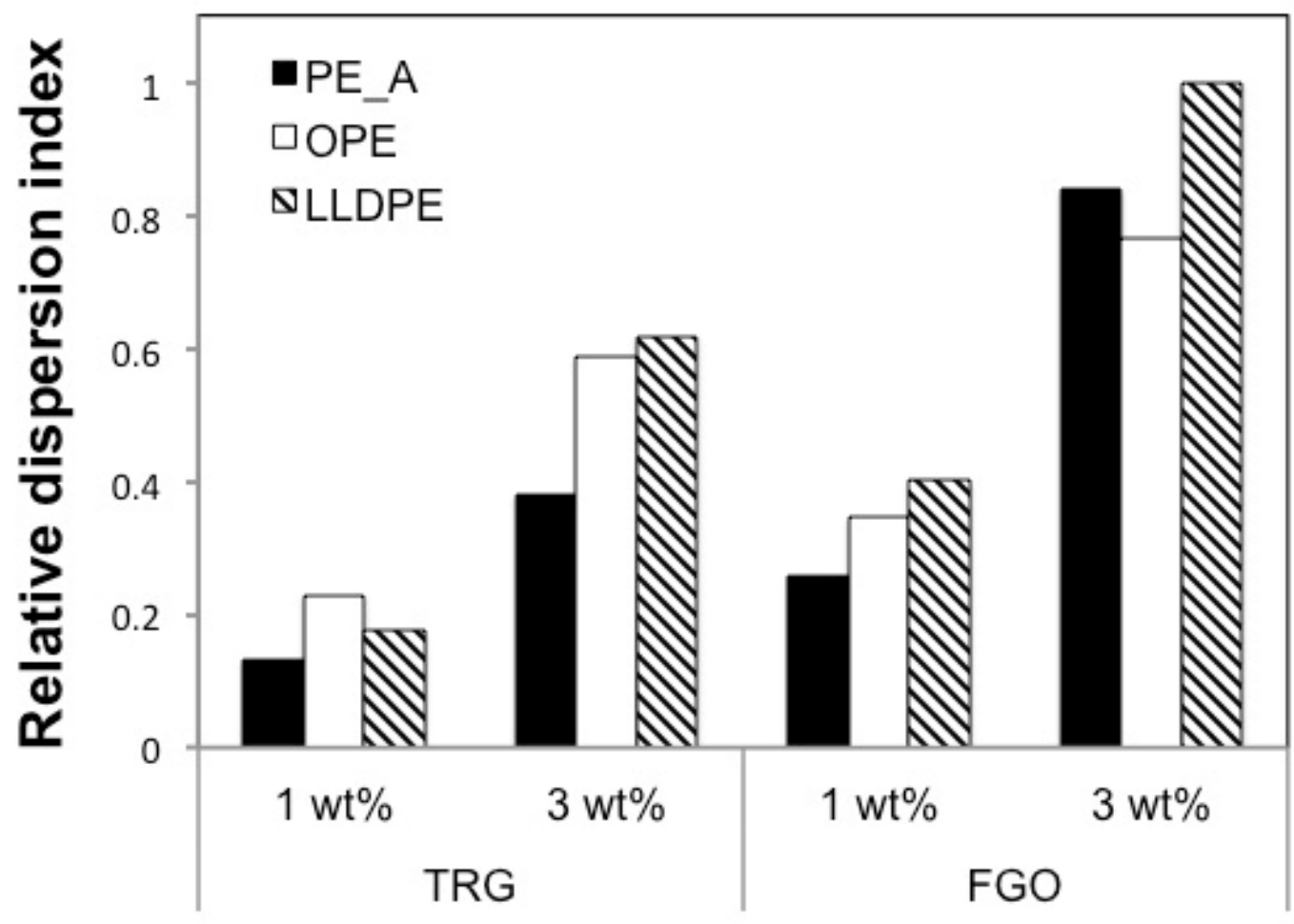


Fig. 6. Optical microscopic images of LLDPE with (a) $3 w t \%$ TRG and (b) $3 w t \%$ FGO. Images were thresholded using ImageJ. (c) Relative dispersion index of $1 \mathrm{wt} \%$ and $3 \mathrm{wt} \%$ of TRG and FGO in PEs. Values are scaled to FG) with PE-A.

\subsection{Properties of nanocomposites}

PE can become electrically conductive and more mechanically robust by graphene reinforcement. Results of electrical resistance and tensile modulus measurements are summarized in Table S2 (see Supplementary Material).

\subsubsection{Electrical conductivity}

The surface resistance of $3 \mathrm{wt} \% \mathrm{FGO}, \mathrm{TRG}$, and $\mathrm{GO}$ in PE after $1 \mathrm{~h}$ annealing is compared in Figure 7a. Even at the same filler concentration, the surface resistance differs considerably depending on graphene and PE compatibility. FGO composites with PE_A and LLDPE revealed substantially improved electrical conductivity. Figure $7 \mathrm{~b}$ and $7 \mathrm{c}$ summarize surface resistance of composites as a function of graphene loading. While TRG/PE composites do not become electrically conductive up to $5 \mathrm{wt} \%$ of TRG, FGO incorporation into PE_A or LLDPE leads to a great decrease in electrical resistance. A negative influence of PE oxidation on conductivity was detected from FGO/OPE composites. Sample spanning pathways for electrical conduction are formed via percolation of graphene in the polymer matrix. Dispersion of graphene in PE inferred from conductivity enhancement generally correlated with the morphological characterization by optical microscopy seen in Figure 6. However, even with seemingly better dispersion from optical images (Fig. 6c and Fig. S4) the decrease in resistance 
for FGO in OPE was only observed above $3 \mathrm{wt} \%$. This is likely due to the abundant oxidized groups on PE hindering the electron transport through graphene sheets in the composites $[27,28]$.

a
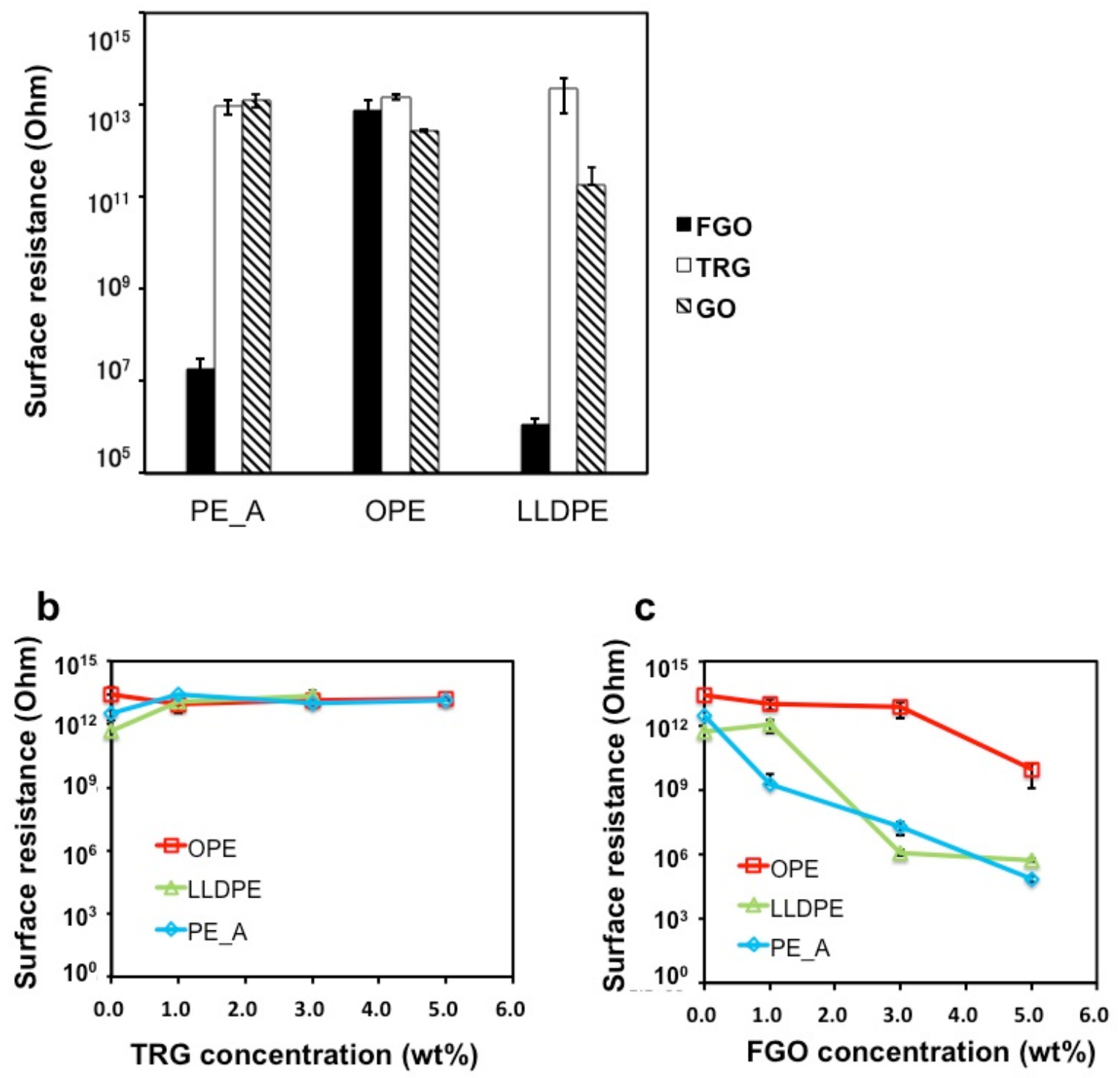

Fig. 7. Surface resistance after $1 \mathrm{~h}$ annealing at $210{ }^{\circ} \mathrm{C}$ of (a) $3 \mathrm{wt} \%$ graphenes in PE matrices, and PE matrices with different concentrations of (b) TRG and (c) FGO. 


\subsubsection{Effect of thermal reduction on electrical conductivity}

The most economical route to produce large quantities of graphene sheets for polymer composites appears to be oxidizing graphite to $\mathrm{GO}[12,13,14,17,19]$. This oxidation step gives rise to a loss of electric conductivity, thus a reduction has to be performed to recover conductivity in PE/graphene nanocomposites. Different approaches, such as thermal treatment [29,30,31,32], chemical reduction [31], and UV irradiation [33,34] have been employed to reduce GO. In this study, thermal reduction was achieved in-situ by heating the nanocomposites.

Generally, temperatures above $600{ }^{\circ} \mathrm{C}$ are needed to perform optimal thermal reduction [29,30,31]. However, the practical temperature of in-situ thermal reduction is restricted by the degradation temperature of polymers [31]. Hence, simple thermal reduction of the FGO sheets dispersed in $\mathrm{PE}$ at moderate temperature, $210^{\circ} \mathrm{C}$, was employed to partially reduce $\mathrm{FGO}$ and to obtain reasonable electrical conductivity without any damage to the functional grafts on FGO or the PE matrices. In order to identify the time required for thermal reduction, FGO/LLDPE samples were treated at $210{ }^{\circ} \mathrm{C}$ for $5 \mathrm{~min}, 30 \mathrm{~min}$, and $1 \mathrm{~h}$ (Fig. S6). The reduction of GO started to occur even after only $5 \mathrm{~min}$, leading to the restoration of electrical conductivity of FGO/LLDPE composites. As the annealing time increased, the electrical conductivity of GO in PE matrix improved further until $1 \mathrm{~h}$. In case of $5 \mathrm{wt} \% \mathrm{FGO}, 5$ min reduction was already enough to obtain FGO/LLPDPE composites with good electrical conductivity. As the annealing time increased, the electrical conductivity of the composites improved further until $1 \mathrm{~h}$. Therefore, $1 \mathrm{~h}$ annealing was selected as the condition of GO reduction for the composites. As expected, Figure 8 shows that the surface resistance of FGO/LLDPE and FGO/PE_A with increasing FGO concentration. There was no change in the as-cast samples but an obvious decrease in resistance after $1 \mathrm{~h}$ annealing at $210{ }^{\circ} \mathrm{C}$. The dramatic change in electrical conductivity was achieved at an FGO content of $3 \mathrm{wt} \%$ because at this loading FGO reached a 
percolation threshold through uniform dispersion within the PE matrix. The high viscosity of the polymer matrix surrounding FGO helped to prevent reaggregation of reduced FGO in the composites $[29,35]$.
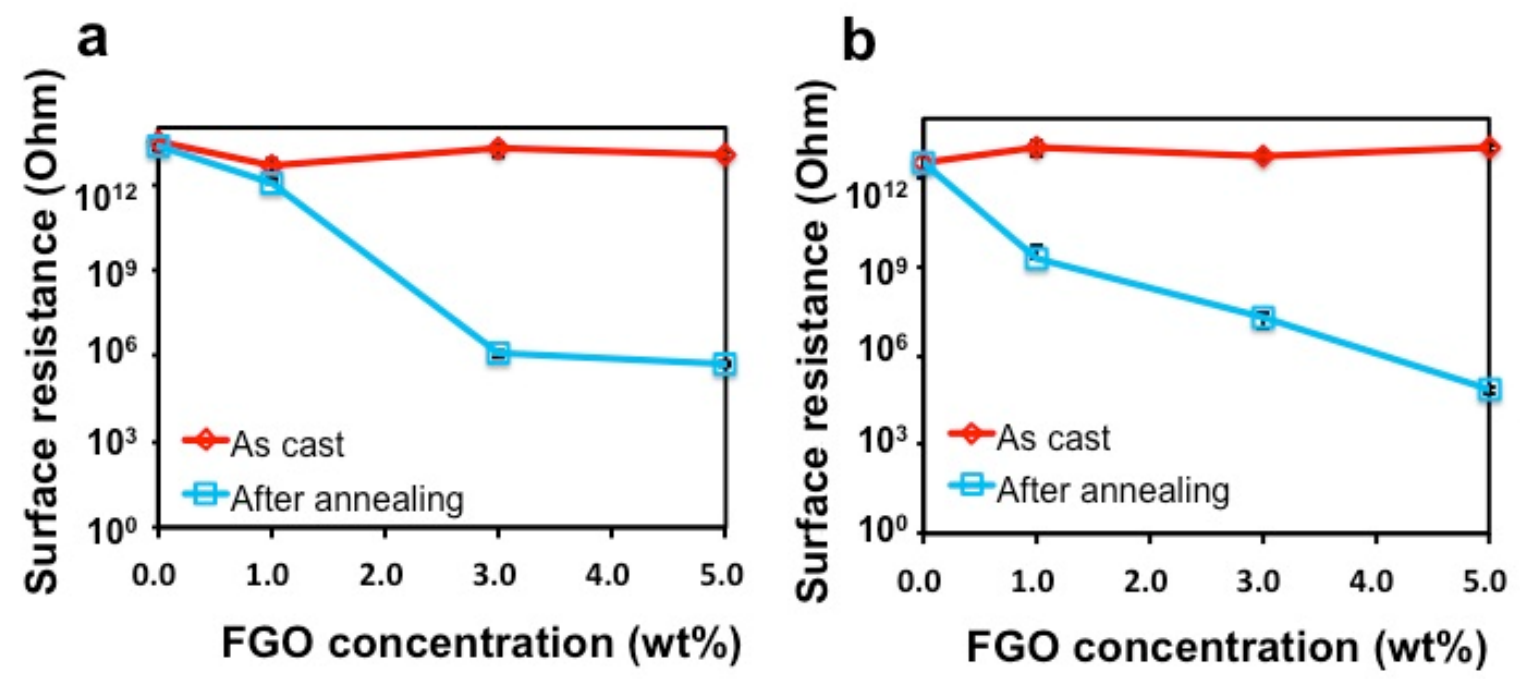

Fig. 8. Surface resistance of (a) LLDPE and (b) PE_A with different FGO concentrations, comparing as-cast samples with those that had been annealed for $1 \mathrm{~h}$.

\subsubsection{Tensile modulus}

Mechanical properties of the PE polymers in this study were improved considerably via dispersion of graphene. At the same graphene concentration ( $5 \mathrm{wt} \%$ ), the tensile moduli depend on the compatibility between the filler and matrices (Fig. 9). Tensile moduli are enhanced significantly by blending with FGO in all types of PE. The highest modulus was achieved with the FGO/PE_A composite (480 MPa). TRG and GO showed less increase in all PE's and even a slight modulus decrease with OPE. 


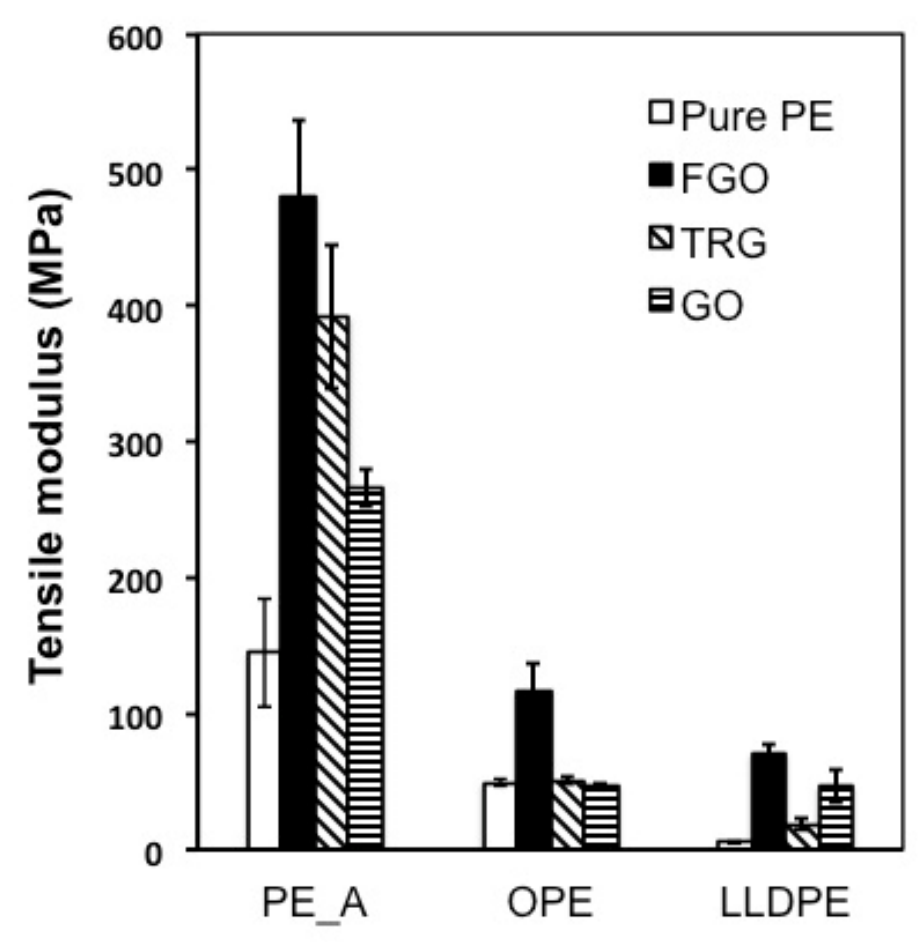

Fig. 9. Tensile moduli of graphene/PE nanocomposites with $5 \mathrm{wt} \%$ filler.

Tensile moduli normalized by modulus $\mathrm{E}_{0}$ of unfilled PE are plotted in Figure 10. Graphene/LLDPE and graphene/PE_A composites show an overall uniform increase in accordance with the graphene composition, whereas the trend is less uniform for the PE composite with GO. Particularly, GO does not show any consistent increase in the normalized tensile moduli with graphene concentration in all PEs due to the poor dispersion in PE caused by the significant hydrophilicity of unfunctionalized GO. Another point to note is that, although OPE shows a good dispersion with all graphenes, the graphenes have little effect on its modulus. We attribute this result to relatively low molecular weight, viscosity (shown in Table 1) and oxide groups of OPE, leading to unstable and heterogeneous composite films, with a cocontinuous morphology of graphene-rich and graphene-poor phases through the repeated thermal treatment. In measuring the tensile modulus we strained all samples up to $4 \%$ strain. OPE composites all 
broke (see Table S3). PE_A and LLDPE composites do not show failure up to $4 \%$ strain indicating their greater ductility. 
a

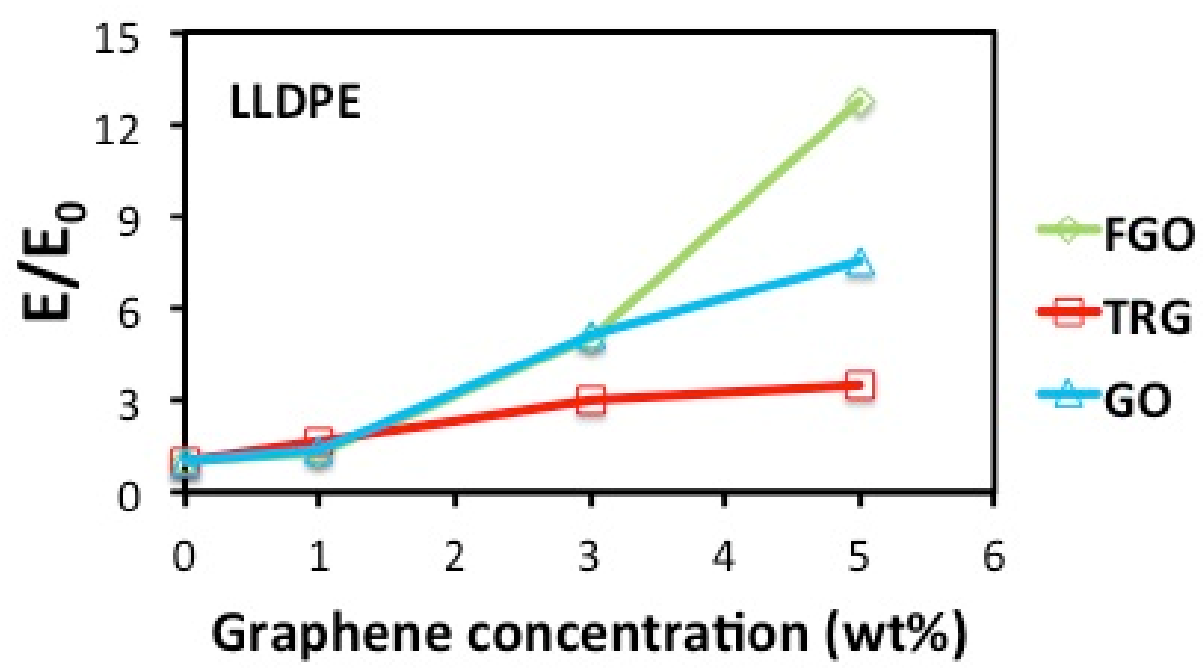

b

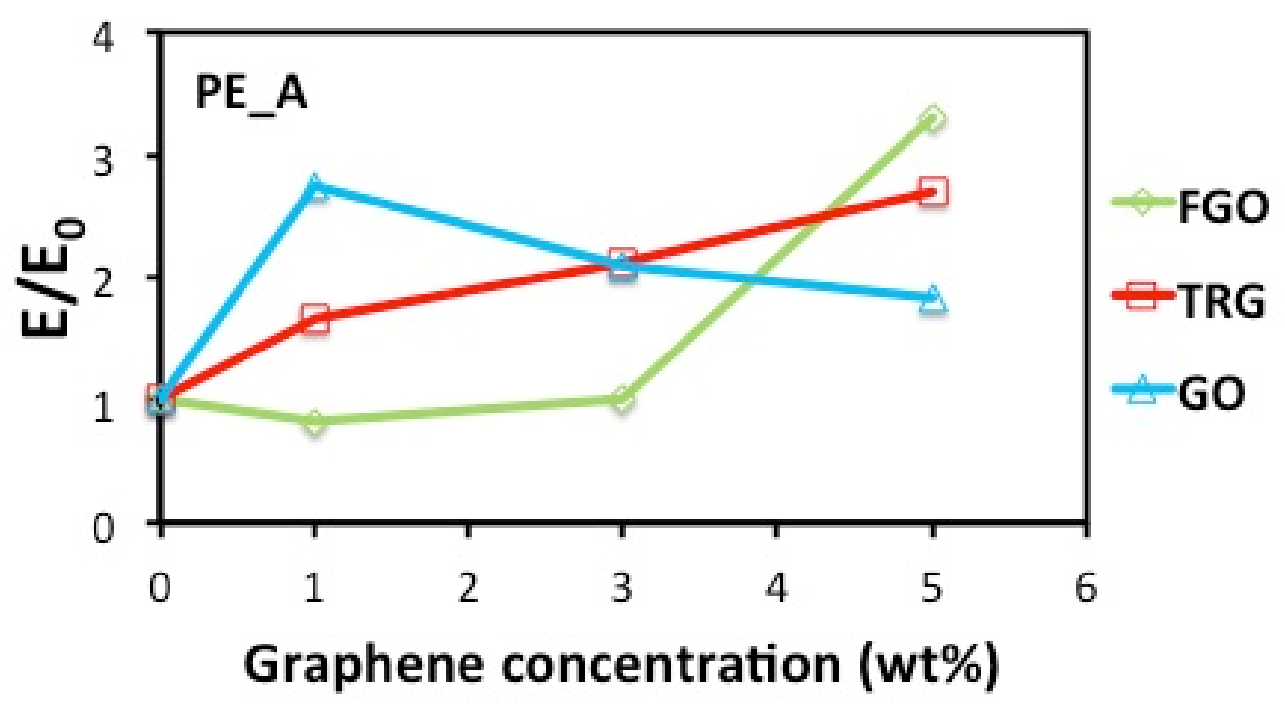

C

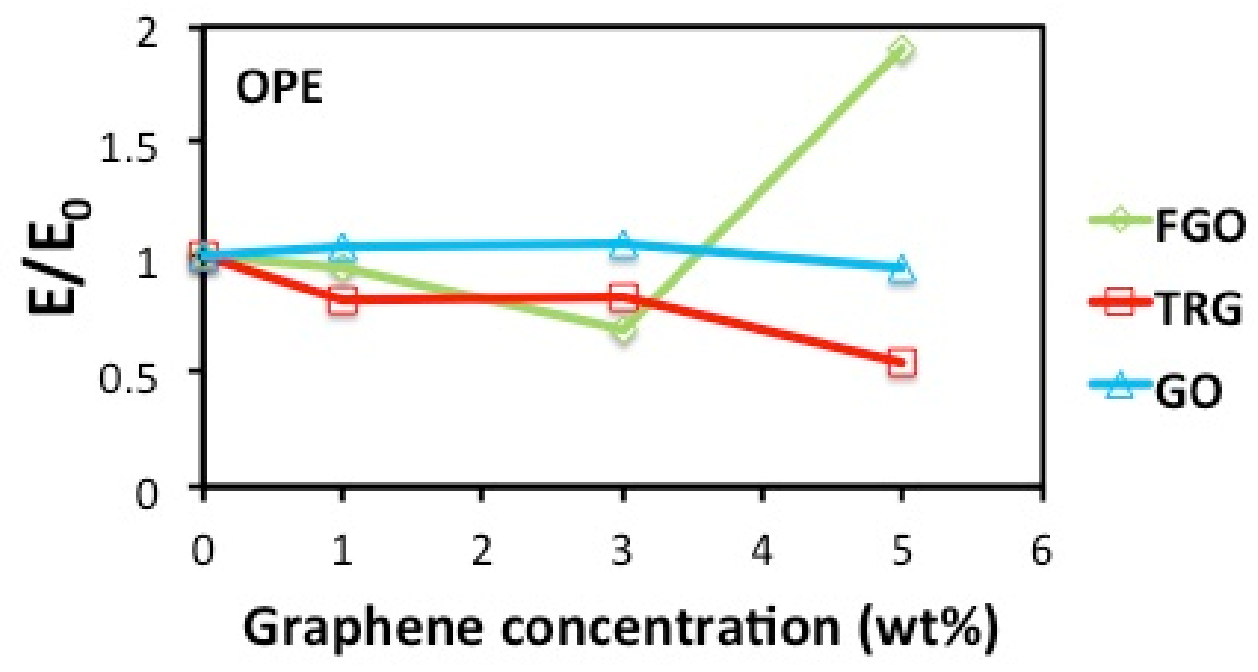


Fig. 10. Tensile modulus of (a) graphene/LLDPE composites, (b) graphene/PE_A composites and (c) graphene/OPE composites.

\section{Conclusions}

In this study, we dispersed GO into three PEs: linear low density LLDPE, high density PE_A, and an oxidized PE (OPE) via solvent blending. Dispersion of GO can be difficult when the matrix is as highly hydrophobic and non-polar as PE. In order to improve its miscibility, GO was functionalized with 18-carbon alkyl chains and benzyl moieties. This FGO was blended into the PE matrices and composite films were obtained by casting from the solution. Graphene/PE composites were also produced from a thermally reduced GO (TRG) and unfunctionalized GO using solvent blending. Even at the same loading, nanocomposite properties strongly depend on spatial distribution of the anisotropic inclusion. Visual observation of cast films revealed a more homogeneous distribution of FGO in PE than GO or TRG due to compatibility between FGO and PE.

A simple thermal treatment of the $\mathrm{FGO}$ nanocomposites at moderate temperature $\left(210^{\circ} \mathrm{C}\right)$ following solvent casting resulted in greatly enhanced electrical conductivity. We achieved surface resistance of composites over $10^{5} \mathrm{ohm}$ at a FGO content of $5 \mathrm{wt} \%$. This thermal treatment yielded an improvement similar to what was observed for poly(methyl methacrylate)/GO [31] and TRG/PE nanocomposites [16] but did not improve conductivity for our TRG or GO/PE composites. This simple process recovered electrical conductivity at moderate temperature without any additional environmental control such as a nitrogen atmosphere. It also appeared to be almost as effective as the chemical reduction with hydrazine [31]. 
Regardless of PE types, FGO increased the tensile modulus of each PE more than that of TRG. The modulus increased $12 \times$ for LLDPE and $3 \times$ for PE_A with $5 \mathrm{wt} \%$ FGO. Enhancements in electrical conductivity and tensile modulus were more pronounced for FGO/LLDPE and FGO/PE_A composites than for FGO/OPE. We attribute this result to the oxidization of PE which hinders the electron transfer through graphene sheets even though the oxide groups of OPE help the graphene distribution in PE matrices.

\section{Acknowledgement}

This work was supported by the Petroleum Research Institute of Abu Dhabi through its Joint Polymer Processing Research Program with the University of Minnesota and by a grant from Adama Materials. Parts of this work were carried out at the University of Minnesota Characterization Facility, which receives partial support from the NSF through the MRSEC, ERC, MRI, and NNIN programs. We thank Vorbeck Materials for the TRG sample.

\section{References}

[1] S. Stankovich, D.A. Dikin, G.H.B. Dommett, K.M. Kohlhaas, E.J. Zimney, E.A. Stach, R.D. Piner, S.T. Nguyen, R.S. Ruoff, Graphene-based composite materials, Nature 442 (2006) 282286.

[2] K. Hu, D.D. Kulkarni, I. Choi, V.V. Tsukruk, Graphene-polymer nanocomposites for structural and functional applications, Prog. Polym. Sci. 39 (2014) 1934-1972.

[3] H. Kim, A.A. Abdala, C.W. Macosko, Graphene/polymer nanocomposites, Macromolecules 43 (2010) 6515-6530.

[4] W. Zhang, A.A. Dehghani-Sanij, R.S. Blackburn, Carbon based conductive polymer composites, J. Mater. Sci. 42 (2007) 3408-3418. 
[5] J.N. Aneli, L.M. Khananasvili, G.E. Zaikov, Structuring and conductivity of polymer composites, New York: Nova Science Publishers (1998).

[6] J. Li, P.C. Ma, W.S. Chow, C.K. To, B.Z. Tang, J.K. Kim, Correlations between percolation threshold, dispersion state and aspect ratio of carbon nanotube, Adv. Funct. Mater. 17 (2007) $3207-3215$.

[7] P.C. Ma, J.K. Kim, B.Z. Tang, Effects of silane functionalization on the properties of carbon nanotubes/epoxy nanocomposites, Compos. Sci. Technol. 67 (2007) 2965-2972.

[8] P.C. Ma, B.Z. Tang, J.K. Kim, Effect of CNT decoration with silver nanoparticles on electrical conductivity of CNT-polymer composites, Carbon 46 (2008) 1497-1505.

[9] W. Bauhofer, J.Z. Kovacs, A review and analysis of electrical percolation in carbon nanotube polymer composites, Compos. Sci. Technol. 69 (2009) 1486-1498.

[10] M.J. Biercuk, M.C. Llaguno, M. Radosavljevic, J.K. Hyun, A.T. Johnson, J.E. Fischer, Carbon nanotube composites for thermal management, Appl. Phys. Lett. 80 (2002) 2767-2769.

[11] L. Zhang, S.D. Allen, C. Woelfle, F. Zhang, Influence of polymer structures on optical power limiting performance of single-walled carbon nanotubes, J. Phys. Chem. C, 113 (2009) $13979-13984$.

[12] S. Park, R.S. Ruoff, Chemical methods for the production of graphenes, Nat. Nanotechnol. 4 (2009) 217-224.

[13] D. Li, M.B. Muller, S. Gilje, R.B. Kaner, G.G. Wallace, Processable aqueous dispersions of graphene nanosheets, Nat. Nanotechnol. 3 (2008) 101-105.

[14] Y. Geng, S.J. Wang, J.K. Kim, Preparation of graphite nanoplatelets and graphene sheets, J. Colloid. Interface Sci. 336 (2009) 592-598.

[15] A.A. Vasileiou, M. Kontopoulou, A. Docoslis, A Noncovalent compatibilization approach to improve the filler dispersion and properties of polyethylene/graphene composites, ACS Appl. 
Mater. Interfaces 6 (2014) 1916-1925.

[16] H. Kim, S. Kobayashi, M.A. AbdurRahim, M.J. Zhang, A. Khusainova, M.A. Hillmyer, A.A. Abdala, C.W. Macosko, Graphene/polyethylene nanocomposites: effect of polyethylene functionalization and blending methods, Polymer 52 (2011) 1837-1846.

[17] W.S. Hummers, R.E. Offeman, Preparation of graphite oxide. J. Am. Chem. Soc., 80 (1958) 1339-1339.

[18] D. Zheng, G. Tang, H.B. Zhang, Z.Z. Yu, F. Yavari, N. Koratkar, S.H. Lim, M.W. Lee, In situ thermal reduction of graphene oxide for high electrical conductivity and low percolation threshold in polyamide-6 nanocomposites, Compos. Sci. Technol. 72 (2012) 284-289.

[19] O.C. Compton, S.T. Nguyen, Graphene oxide, highly reduced graphene oxide, and graphene: versatile building blocks for carbon -based materials, Small 6 (2010) 711-723.

[20] R.D. Dreyer, S. Park, C.W. Bielawski, R.S. Ruoff, The chemistry of graphene oxide, Chem. Soc. Rev. 39 (2010) 228-240.

[21] L. Mandelkern, J.G. Fatou, R. Denison, J. Justin, A calorimetric study of the fusion of molecular weight fractions of linear polythylene (1), J. Polym. Sci. Part B 3 (1965) 803-807.

[22] H.C. Schniepp, J.L. Li, M.J. McAllister, H. Sai, M. Herrera-Alonso, D.H. Adamson, R.K. Prud'homme, R. Car, D.A. Saville, I.A. Aksay, Functional single graphene sheets derived from splitting graphite oxide, J. Phys. Chem. B. 110 (2006) 8535-8539.

[23] G. Liu, Y. Wang, C. Xu, F.Qiu, C. An, L. Li, L. Jiao, H. Yuan, Excellent catalytic effects of highly crumpled graphene nanosheets on hydrogenation/dehydrogenation of magnesium hydride, Nanoscale 5 (2013) 1074-1081.

[24] R.M. Silvesrstein, G.C. Bassler, T.C. Morrill, Spectrometric identification of organic compounds, $4^{\text {th }}$ ed., John Wiley \& Sons: New York, 1981. 
[25] T. Szabo, O. Berkesi, I. Dekany, DRIFT study of deuterium-exchanged graphite oxide, Carbon 43 (2005) 3181-3194.

[26] H. Kim, Y. Miura, C.W. Macosko, Graphene/polyurethane nanocomposites for improved gas barrier and electrical conductivity, Chem. Mater. 22 (2010) 3441-3450.

[27] Y.D. Lim, D.Y. Lee, T.Z. Shen, C.H. Ra, J.Y. Choi, W.J. Yoo, Si-compatible cleaning process for graphene using low-density inductively coupled plasma, ACS Nano 6 (2012) 44104417.

[28] S. Ryu, L. Liu, S. Berciaud, Y.J. Yu, H. Liu, P. Kim, G.W. Flynn, L.E. Brus, Atmospheric oxygen binding and hole doping in deformed graphene on a $\mathrm{SiO}_{2}$ substrates, Nano Lett. 10 (2010) 4944-4951.

[29] S. Ye, J. Feng, A new insight into the in situ thermal reduction of graphene oxide dispersed in a polymer matrix, J. Polym. Chem. 4 (2013) 1765-1768.

[30] O. Akhavan, The effect of heat treatment on formation of graphene thin films from graphene oxide nanosheets, Carbon 48 (2009) 509-519.

[31] J.M. Thomassin, M. Trifkovic, W. Alkarmo, C. Detrembleur, C. Jerome, C. Macosko, Poly(methyl methacrylate)/graphene oxide nanocomposites by a precipitation polymerization process and their dielectric and rheological characterization, Macromolecules 47 (2014) 21492155.

[32] W. Chen, L. Yan, Preparation of graphene by a low-temperature thermal reduction at atmosphere pressure, Nanoscale 2 (2010) 559-563.

[33] H. Li, S. Pang, S. Wu, X. Feng, K. Mullen, C. Bubeck, Layer-by-layer assembly and UV photoreduction of graphene-polyoxometalate composite films for electronics, J. Am. Chem. Soc. 133, (2011) 9423-9429. 
[34] G. Williams, B. Seger, P.V. Kamat, $\mathrm{TiO}_{2}$-graphene nanocomposites. UV-assisted photocatalytic reduction of graphene oxide, ACS Nano 2 (2008) 1487-1491.

[35] M. Traina, A. Pegoretti, In situ reduction of graphene oxide dispersed in a polymer matrix, J. Nanopart. Res. 14 (2012) 801/1-6. 